\title{
Laser-cut-type versus braided-type covered self-expandable metallic stents for distal biliary obstruction caused by pancreatic carcinoma: a retrospective comparative cohort study
}

\author{
Koh Kitagawa, Akira Mitoro, Takahiro Ozutsumi, Masanori Furukawa, Yukihisa Fujinaga, Kenichiro Seki, Norihisa Nishimura, \\ Yasuhiko Sawada, Kosuke Kaji, Hideto Kawaratani, Hiroaki Takaya, Kei Moriya, Tadashi Namisaki, Takemi Akahane, \\ Hitoshi Yoshiji
}

Department of Gastroenterology, Nara Medical University, Nara, Japan

\begin{abstract}
Background/Aims: Covered self-expandable metallic stents (CMSs) are widely used for malignant distal biliary obstructions (MDBOs) caused by pancreatic carcinoma. This study compared the efficacy and safety of the laser-cut-type and braided-type CMSs.

Methods: To palliate MDBOs caused by pancreatic carcinoma, the laser-cut-type CMSs was used from April 2014 to March 2017, and the braided-type CMSs was used from April 2017 to March 2019. The tested self-expandable metallic stents were equipped with different anti-migration systems.

Results: In total, 47 patients received CMSs for MDBOs (24 laser-cut type, 23 braided-type). The time to recurrent biliary obstruction (TRBO) was significantly longer in the braided-type CMSs $(p=0.0008)$, and the median time to stent dysfunction or patient death was 141 and 265 days in the laser-cut-type CMSs and braided-type CMSs, respectively $(p=0.0023)$. Stent migration was the major cause of stent dysfunction in both groups, which occurred in 37.5\% of the laser-cut-type CMSs and 13.0\% of the braided-type CMSs. There were no differences in the survival duration between the groups.

Conclusions: The TRBO was significantly longer for the braided-type CMSs with an anti-migration system than for the laser-cut-type. Stent migration tended to be less frequent with the braided-type CMSs than with the laser-cut-type CMSs.
\end{abstract}

Keywords: Adverse effects; Biliary tract; Self-expandable metallic stents

\section{INTRODUCTION}

Malignant distal biliary obstruction (MDBO) is a common

Received: May 17, 2021 Revised: July 10, $2021 \quad$ Accepted: July 11, 2021

Correspondence: Koh Kitagawa

Department of Gastroenterology, Nara Medical University, 840 Shijo-cho, Kashihara, Nara 634-8522, Japan

E-mail: kitagawa@naramed-u.ac.jp

Correspondence: Akira Mitoro

Department of Gastroenterology, Nara Medical University, 840 Shijo-cho, Kashihara, Nara 634-8522, Japan

E-mail: mitoroak@naramed-u.ac.jp

(a) This is an Open Access article distributed under the terms of the Creative Commons Attribution Non-Commercial License (http://creativecommons.org/licenses/by-nc/4.0/) which permits unrestricted non-commercial use, distribution, and reproduction in any medium, provided the original work is properly cited. complication in patients with pancreatic carcinoma. Endoscopic stent placement is the treatment of choice for palliation of MDBO. ${ }^{1-4}$ Several types of stents, including plastic stents, uncovered self-expandable metallic stents (SEMSs), and covered SEMSs (CMSs), are available for use in clinical practice. It should be noted that the usefulness of SEMSs has been widely reported since the $1980 \mathrm{~s}^{5}$ In particular, in patients with unresectable malignant biliary obstruction, SEMSs are considered to have better patency, larger diameters, and are more cost-effective than plastic stents. ${ }^{6-10}$ However, conventional uncovered SEMSs (UMSs) are prone to occlusion, mainly by tumor ingrowth through the mesh. To overcome this issue, CMSs, in which the stent mesh is covered with a thin membrane, have been developed. In some randomized controlled trials, CMSs 
have been reported to be more useful than UMSs in terms of longer patency; ${ }^{11,12}$ however, on the other hand, CMSs are associated with specific complications, such as sludge formation ${ }^{13-15}$ and stent migration. ${ }^{16-21}$ In particular, migration is an important issue because CMSs are not embedded in the bile duct. Conio et al. ${ }^{20}$ reported that CMSs had worse patency than UMSs; therefore, it remains controversial whether UMSs or CMSs are more useful. ${ }^{17,19,21-24}$ Recently, several types of CMSs with anti-migration systems have been developed to prevent stent migration. ${ }^{25-28}$

There are two types of CMSs: laser-cut-type and braided-type. Differences in the manufacturing process may affect mechanical properties, such as the axial force (AF), which is a straightening force, or the radial force (RF), which is an expanding force. ${ }^{29}$ It is unclear how the mechanical differences in CMSs affect stent patency and the incidence of adverse events (AEs), as well as the superiority of the CMSs type.

To the best of our knowledge, there have been no direct comparisons of the laser-cut-type CMSs and braided-type CMSs reported in the literature. This study aimed to assess differences in stent patency and safety between the laser-cut-type CMSs and braided-type CMSs. Considering that the critical problem of CMSs is stent migration, we decided to conduct the study using CMSs equipped with anti-migration systems.

\section{METHODS}

Figure 1 shows the patient flowchart of this study. Fifty-one patients underwent initial SEMS placement for MDBO caused by pancreatic carcinoma between April 2014 and March 2019 at our institution. The diagnosis of MDBO was made based on pathological and/or radiological findings and/or clinical courses. Data before stent insertion were also analyzed, including patient age, sex, the World Health Organization (WHO) performance status, tumor size, presence of distant metastasis or duodenal invasion, length of biliary stricture, total bilirubin, and the presence of prior plastic stent drainage or endoscopic nasobiliary drainage.

We performed endoscopic biliary stenting using laser-cuttype CMSs (ZEOSTENT covered; Zeon Medical, Tokyo, Japan) from April 2014 to March 2017. The total number of SEMSs placements for MDBO caused by pancreatic carcinoma in these 3 years was 24 . All 24 patients with ZEOSTENT CMSs placements were assigned to the laser-cut group. We used the braided-type CMSs (WallFlex Biliary RX Partially Covered Stent System; Boston Scientific, Marlborough, MA, USA) from April 2017 to March 2019. The total number of SEMSs placements for MDBO caused by pancreatic carcinoma in these 2 years was 27. Of these, three patients who underwent the laser-cut-type CMS placement because of technical difficulties when placing the braided-type CMSs were excluded from the study. Another patient who received an uncovered SEMS because of pancreatitis on the previous endoscopic retrograde cholangiopancreatography (ERCP) with a plastic stent was also excluded. The remaining 23 patients with a partially covered WallFlex stent were included in the braided group. Finally, 47 patients (la-

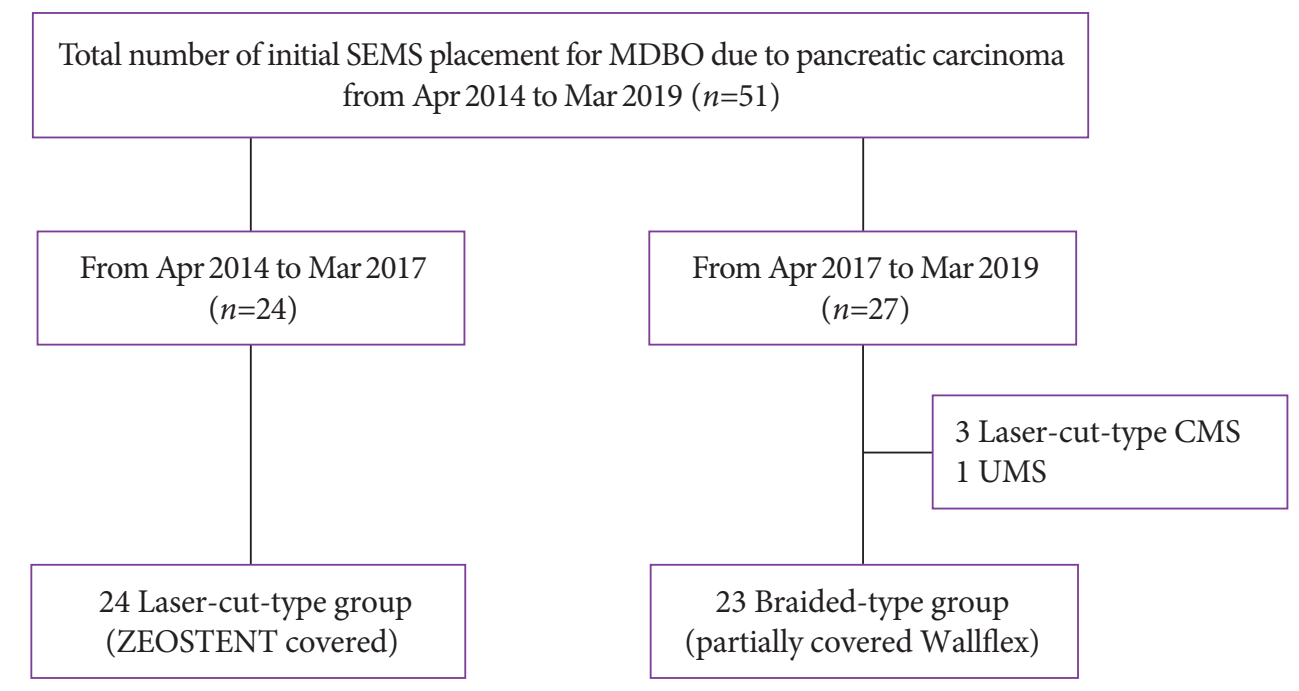

Fig. 1. Patient flowchart of the study. SEMS, self-expandable metallic stent; MDBO, malignant distal biliary obstruction; CMS, covered self-expandable metallic stent; UMS, uncovered self-expandable metallic stent. 
ser-cut group, $n=24$; braided group, $n=23$ ) were enrolled in the retrospective cohort.

\section{Procedure}

All procedures were performed using a standard therapeutic duodenoscope (JF260V/TJF260V; Olympus Optical, Tokyo, Japan) under fluoroscopic guidance. After selective bile duct cannulation, cholangiography was performed, and the bile duct stricture length was measured. A CMS was placed at the biliary stricture during the ERCP. Endoscopic sphincterotomy (ES) was performed at the discretion of the endoscopist. The diameter of the stent was $8 \mathrm{~mm}$ or $10 \mathrm{~mm}$, and the length of the stent was $60 \mathrm{~mm}, 70 \mathrm{~mm}$, or $80 \mathrm{~mm}$. Stents with a length of $70 \mathrm{~mm}$ were not available in the braided-type CMSs. The length and diameter of the stents were determined according to the length of the biliary stricture or the diameter of the bile duct.

The distal end of the CMSs was positioned below the papilla in all patients. In this study, none of the patients received rectal indomethacin during the periprocedural period.

\section{Endpoints and definitions}

The primary endpoint of our study was time to recurrent biliary obstruction (TRBO) for the laser-cut-type CMSs and braided-type CMSs for palliation of patients with MDBO caused by pancreatic carcinoma. Secondary endpoints were early and late $\mathrm{AE}$ rates, patient overall survival (OS) duration, and causes of stent dysfunction.

All the terms used in this study were those used in the TOKYO criteria 2014 for transpapillary biliary stenting. ${ }^{30} \mathrm{Re}-$ current biliary obstruction (RBO) was defined as the point at which clinical features associated with occlusion or migration were observed. TRBO was defined as the period between initial metallic stent placement and RBO, and the OS duration was defined as the time between initial metallic stent placement and death or the last follow-up. AE other than RBO were also defined according to the TOKYO criteria $2014 .^{30}$

AEs were classified as early AEs when they occurred within 14 days of the procedure and as late AEs when they occurred after 14 days of the procedure. AE severity was graded according to the American Society of Gastrointestinal Endoscopy lexicon. ${ }^{31}$

Assessment of the change in tumor burden due to chemotherapy was based on the Revised Response Evaluation Criteria in Solid Tumors guidelines (version 1.1). ${ }^{32}$ Tumor burden was determined using computed tomography scanning 2-3 months after CMS placement. Patients who died within 2 months of stent placement were considered to have progressive disease.

\section{Statistical analysis}

The chi-squared test or Fisher exact test was used to compare categorical variables, and the $t$-test or Mann-Whitney $U$-test was used to compare continuous variables. Statistical significance was set at $p<0.05$. TRBO and OS were estimated using the Kaplan-Meier method and were compared between the laser-cut and braided groups using the log-rank test. The TRBO and OS for patients who had not experienced RBO or death were censored at the end of each patient's follow-up point. All statistical analyses were performed using the EZR ver. 1.41 (Saitama Medical Center, Jichi Medical University, Saitama, Japan), which is a graphical user interface for R ver. 4.0.3 (The R Foundation for Statistical Computing, Vienna, Austria). ${ }^{33}$

\section{Ethical statements}

This study was approved by the Institutional Review Board of Nara Medical University Hospital (IRB No: 2267).

\section{RESULTS}

\section{Patient characteristics}

A total of 47 patients (24 in the laser-cut group and 23 in the braided group) were included in this study. The baseline characteristics are presented in Table 1. Other than age, there were no significant differences in the other patient demographics, including sex, the WHO performance status, tumor size, the presence of distant metastasis or duodenal invasion, length of biliary stricture, total bilirubin, and the presence of prior drainage between the laser-cut-type and braided-type groups. The proportion of patients receiving chemotherapy before and after CMS placement was also not significantly different between the laser-cut and braided groups.

\section{Endoscopic procedure and AEs}

The endoscopic procedures and AEs are presented in Table 2. In the laser-cut group, one patient received an 8-mm diameter stent, and the remaining 23 patients received $10-\mathrm{mm}$ diameter stents. In the braided group, all 23 patients received $10-\mathrm{mm}$ diameter stents. Stents measuring $6 \mathrm{~cm}$ in length were more often used in the braided group; however, 7-cm long stents were more often used in the laser-cut group. ES was performed in three patients in the laser-cut group and two patients in the braided 
Table 1. Patient characteristics

\begin{tabular}{lccc}
\hline Characteristic & Laser-cut-type & Braided-type & p-value \\
\hline Total & 24 & 23 & 0.007 \\
Age (yr) & $71(50-87)$ & $165-90)$ & 0.772 \\
Sex (male:female) & $12: 12$ & $9: 13: 1$ & 0.343 \\
WHO performance status (0:1:2) & $12: 9: 3$ & $31(18-40)$ & 0.316 \\
Tumor size (mm) & $26.5(13-85)$ & $7(30.4)$ & 0.547 \\
Distant metastasis & $10(41.7)$ & $5(21.7)$ & 0.740 \\
Duodenal invasion & $7(29)$ & $22(10-36)$ & 0.436 \\
Length of biliary stricture (mm) & $20(10-46)$ & $2.00(0.8-10.4)$ & 0.406 \\
Serum bilirubin before stenting (mg/dL) & $2.25(0.3-14.7)$ & $16(69.6)$ & 0.238 \\
Prior biliary drainage by PS or ENBD & $12(50)$ & $17(73.9)$ & 0.286 \\
Chemotherapy & $21(87.5)$ & $2(8.7)$ & $15(65.2)$ \\
$\quad$ GEM or S1 & $8(33.3)$ & 0 & 0.516 \\
GnP or mFFX & $12(50.0)$ & $261(28-765)$ & \\
Others & $1(4.2)$ & & \\
Follow-up (day) & $187(55-1,052)$ & & \\
\hline
\end{tabular}

Values are presented as median (range) or number (\%).

WHO, World Health Organization; PS, plastic stent; ENBD, endoscopic nasobiliary drainage; GEM, gemcitabine; GnP, gemcitabine plus nab-paclitaxel; mFFX, modified FOLFIRINOX (fluorouracil, leucovorin, irinotecan, and oxaliplatin).

group, and there was no significant difference in the percentage of patients requiring ES. None of the patients underwent pancreatic drainage.

Mild acute pancreatitis, as an early AE, occurred in two patients (8.7\%) in the braided group. Acute cholecystitis, as a late $\mathrm{AE}$, occurred in one patient $(4.2 \%)$ in the laser-cut group and two patients $(8.7 \%)$ in the braided group. One patient belonging to the laser-cut group had mild cholecystitis. Two patients in the braided group had moderate cholecystitis, stent removal was performed in one patient, and percutaneous transhepatic gallbladder drainage was performed in the other patient. The severity of cholecystitis was graded according to the Tokyo Guidelines 2018. ${ }^{34}$ There were no procedure-related severe AEs or mortality.

\section{Patient survival}

The median follow-up period was 187 days (range, 55-1,052 days) in the laser-cut group and 261 days (range, 28-765 days) in the braided group $(p=0.516)$. The Kaplan-Meier curve for patient survival is shown in Figure 2. The median survival was 187 days (95\% confidence interval [CI], 140-273 days) in the laser-cut group and 269 days (95\% CI, 169-381 days) in the braided group. There were no significant differences in the OS duration between the laser-cut and braided groups $(p=0.618)$.
Table 2. Endoscopic procedure and adverse events

\begin{tabular}{lccc}
\hline Variable & Laser-cut-type & Braided-type & p-value \\
\hline Total & 24 & 23 & \\
Stent diameter $(8 \mathrm{~mm}: 10 \mathrm{~mm})$ & $1: 23$ & $0: 23$ & 1.00 \\
Median stent length $(\mathrm{cm})$ & 7 & 6 & 0.005 \\
Endoscopic sphincterotomy & $3(13)$ & $2(8.7)$ & 1.00 \\
$\begin{array}{l}\text { Pancreatic drainage } \\
\text { Early adverse events }\end{array}$ & 0 & 0 & $\mathrm{NA}$ \\
$\quad$ Pancreatitis & 0 & $2(8.7)$ & 0.234 \\
Late adverse events & & & \\
$\quad$ Cholecystitis & $1(4.2)$ & $2(8.7)$ & 0.609 \\
\hline
\end{tabular}

Values are presented as number (\%).

NA, not applicable.

\section{TRBO}

The Kaplan-Meier curves for TRBO are shown in Figure 3. The median TRBO was 217 days (95\% CI, 125-285 days) in the laser-cut group. In contrast, TRBO analysis could not be performed in the braided group because most patients died by the time stent dysfunction occurred. The median TRBO was significantly longer in the braided-type group with the partially covered WallFlex stent than in the laser-cut group $(p=0.0008)$. Figure 4 shows the median TRBO or patient death. The median TRBO or patient death was also significantly longer in the braided group (265 days; 95\% CI, 126-357 days) than in the laser-cut group (141 days; 95\% CI, 104-203 days; $p=0.023$ ). 


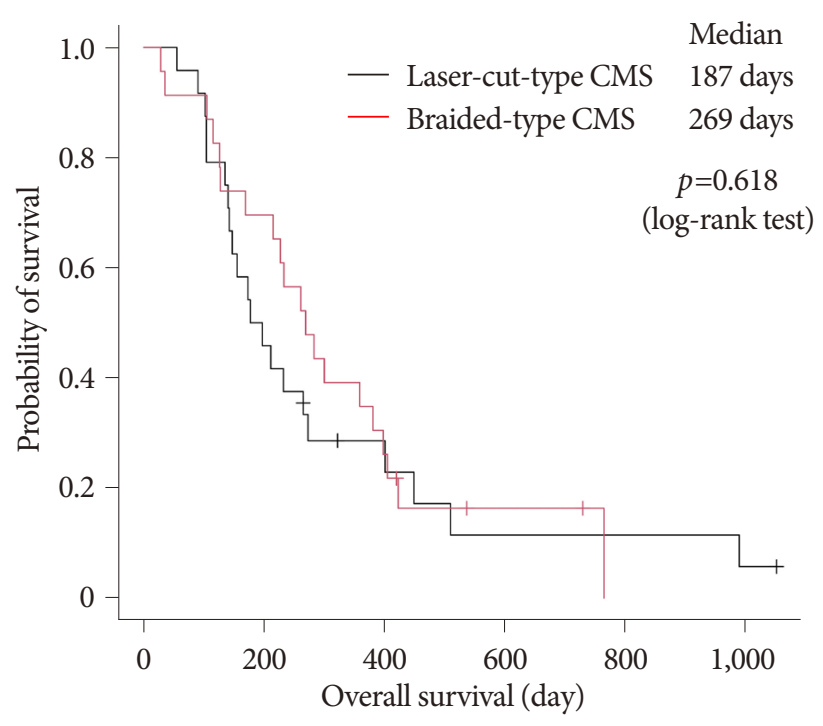

Number at risk

$\begin{array}{llllll}24 & 11 & 5 & 2 & 2 & 1 \\ 23 & 16 & 6 & 2 & 0 & 0\end{array}$

Fig. 2. Kaplan-Meier graph showing the overall survival duration of the patients. No significant difference in the overall survival duration was observed between the laser-cut-type and braided-type CMS groups. CMS, covered self-expandable metallic stent.

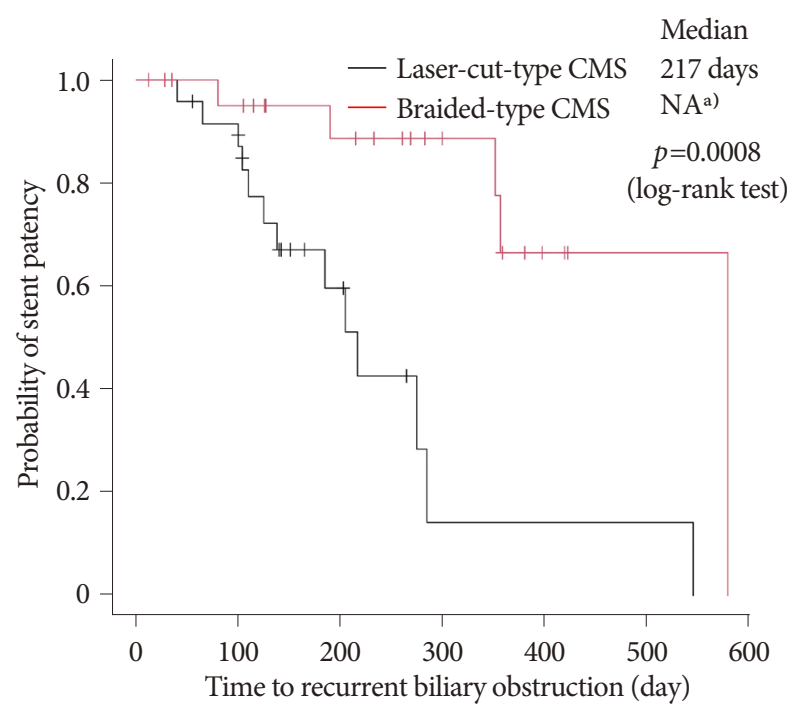

Number at risk

$\begin{array}{lllllll}24 & 21 & 8 & 1 & 1 & 1 & 0 \\ 23 & 19 & 14 & 9 & 3 & 1 & 0\end{array}$

Fig. 3. Kaplan-Meier curve showing the stent patency. Cumulative stent patency was significantly higher for the braided-type CMS than for the laser-cut-type CMS. CMS, covered self-expandable metallic stent. ${ }^{\text {a) }}$ Because most patients died by the time stent dysfunction occurred, the median time for stent patency could not be calculated.

\section{Stent dysfunction}

The results of stent dysfunction are presented in Table 3. Stent dysfunction was observed in 13 patients in the laser-cut group and four patients in the braided group. The rate of stent dysfunction was significantly higher in the laser-cut group than in the braided group $(p=0.015)$. There was a slight trend towards a higher incidence of stent migration in the laser-cut group, although no significant difference was observed. There were no significant differences in the incidence of tumor ingrowth/ overgrowth and sludge formation between the two groups. The anti-cancer effects of chemotherapy and stent length on stent migration are shown in Table 4. In the laser-cut group, successful chemotherapy significantly increased the rate of stent migration, whereas there was no significant difference in the stent length between the groups.

\section{DISCUSSION}

This is the first study to directly compare two different mechanical types of CMSs used with anti-migration systems in patients with $\mathrm{MDBO}$ due to unresectable pancreatic carcinoma. In this

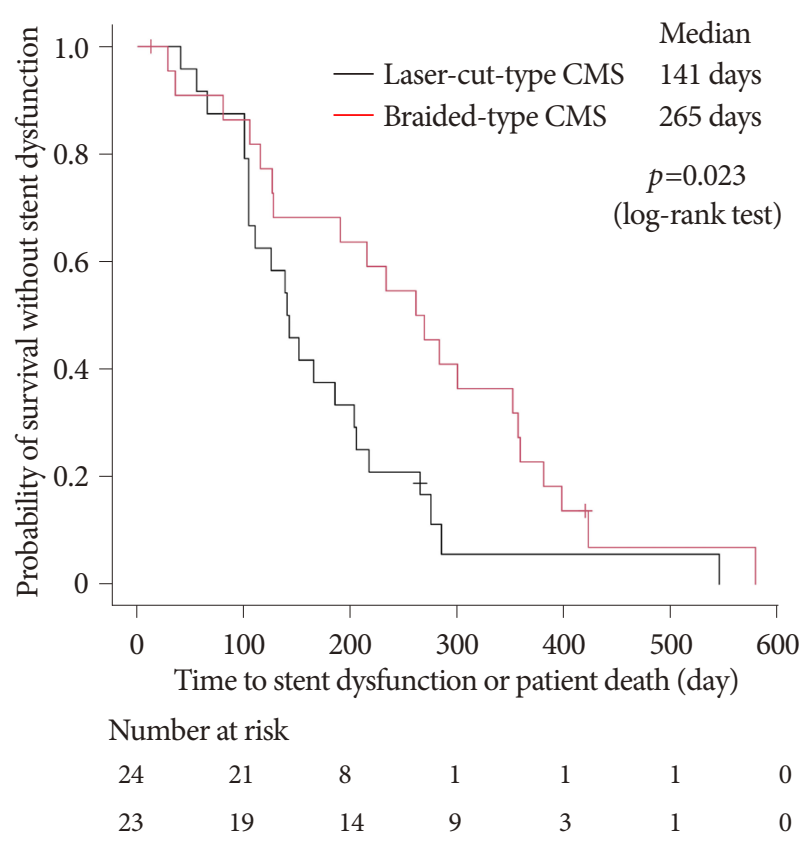

Fig. 4. Kaplan-Meier curve showing the cumulative patient survival without stent dysfunction. The cumulative time to stent dysfunction or patient death was significantly higher in the braided-type CMS group than in the laser-cut-type CMS group. CMS, covered self-expandable metallic stent. 
Table 3. Stent patency duration, dysfunction, and causes in the covered self-expandable metallic stents

\begin{tabular}{lccc}
\hline Variable & Laser-cut-type & Braided-type & p-value \\
\hline Total & 24 & 23 & 0.023 \\
Median to RBO or patient death (day) & 141 & $5(21.7)$ & 0.036 \\
Causes of stent dusfunction & $13(54.2)$ & 0 & 1.000 \\
Tumor ingrowth & $1(4.2)$ & 0 & 0.489 \\
Tumor overgrowth & $2(8.3)$ & $2(8.7)$ & 0.609 \\
Sludge formation & $1(4.2)$ & $3(13.0)$ & 0.093 \\
Stent migration & $9(37.5)$ & & \\
\hline
\end{tabular}

Values are presented as number (\%).

$\mathrm{RBO}$, recurrent biliary occlusion.

Table 4. Factors associated with stent migration

\begin{tabular}{|c|c|c|c|c|c|c|}
\hline \multirow{2}{*}{ Variable } & \multicolumn{3}{|c|}{ Laser-cut-type ( $n=24)$} & \multicolumn{3}{|c|}{ Braided-type $(n=23)$} \\
\hline & Migration absent & Migration present & $p$-value & Migration absent & Migration present & $p$-value \\
\hline Number & $15(62.5)$ & $9(37.5)$ & & $20(87.0)$ & $3(13.0)$ & \\
\hline $\mathrm{BSC}$ or PD & $11(45.8)$ & $2(8.3)$ & & $13(56.5)$ & $2(8.7)$ & \\
\hline $\mathrm{SD}$ or $\mathrm{PR}$ & $4(16.7)$ & $7(29.2)$ & 0.033 & $7(30.4)$ & $1(4.3)$ & 1.000 \\
\hline Median stent length $(\mathrm{cm})$ & 7 & 7 & 0.873 & 6 & 6 & 0.372 \\
\hline
\end{tabular}

Values are presented as number (\%).

$\mathrm{BSC}$, best supportive care; $\mathrm{PD}$, progressive disease; $\mathrm{SD}$, stable disease; $\mathrm{PR}$, partial response.

study, TRBO was shown to be significantly longer in the braided group than in the laser-cut group. In both stent groups, stent migration was the main cause of stent dysfunction; however, there was a trend towards a lower incidence of migration in the braided group, which may have contributed to the difference in TRBO. It was generally expected that laser-cut-type SEMSs, which have a lower AF and higher conformability, would show less migration; however, our results were different. This difference may be related to the differences in the anti-migration systems. Moreover, the laser-cut-type CMSs with less AF and RF and better conformability were thought to be less likely to cause pancreatitis and cholecystitis; however, the frequencies of these complications were similar to those of the braided-type CMSs.

Many types of SEMSs with varying mechanical properties are commercially available. Isayama et al. ${ }^{29}$ investigated the physical properties of various stents in desk experiments and found that braided SEMSs, such as the WallFlex stent, tended to have a relatively strong RF and AF, whereas laser-cut-type SEMSs had a slightly weaker RF and AF, with good conformability. The major advantage of the laser-cut-type SEMSs is that it undergoes less shortening than that of the braided SEMSs, which makes positioning easier. However, there have been no reported comparisons of the effects of these different mechanical properties on stent patency and safety in clinical practice. The ZEOSTENT CMS is made of laser-cut nitinol (nickel-titanium alloy), and the covered membrane comprises polyurethane and polyolefin elastomer. ${ }^{35}$ The outer surface of the stent is covered with a polymer membrane. In addition, this stent is equipped with an anti-migration system. The ZEOSTENT CMS has a "flare and bank" structure, both ends of the stent are flared $(1.5 \mathrm{~cm})$, and a raised band is added at each end, $1 \mathrm{~cm}$ from the $1.5-\mathrm{cm}$ flared portion. On the other hand, the WallFlex Biliary RX stent is partially covered with a single silicone membrane on the inner side of a metallic stent except for a 5-mm section at each end. ${ }^{25}$ Some reports have demonstrated the usefulness of the partially covered WallFlex in MDBO due to pancreatic carcinoma and found that the incidence of stent migration was lower. $^{12,36}$

There have only been a few reports on covered laser-cut-type stents for MDBO. ${ }^{35}$ Isayama et al. ${ }^{35}$ and Marui et al. ${ }^{37}$ reported stent migration rates of $12 \%$ and $4 \%$, respectively. However, the migration rate of the laser-cut-type CMSs remains unclear. Furthermore, few studies have examined whether the anti-migration features of biliary SEMSs prevent migration. Park et al. ${ }^{38}$ compared the anti-migration effects of two types of fully covered metallic stents (FCMSs), concluding that the anchor- 
ing design was superior to the flared-end design. Thus, our tested laser-cut CMSs with a flared design alone may have had a weaker anti-migration effect. Minaga et al. ${ }^{39}$ also investigated the anti-migration properties of several types of FCMSs in desk experiments. In this desk experiment, it was pointed out that there is a strong correlation between the anti-migration effect and RF when the stent is not fully expanded; however, once the stent is fully expanded, the flare structure variables affect the anti-migration properties of the stent. It was also pointed out that laser-cut stents have a strong anti-migration effect when not fully expanded; however, the effect is greatly reduced when the stent is fully expanded. ${ }^{39}$ In our study, we found a significant correlation between response to chemotherapy and increased migration rate, especially in the laser-cut group. The flare and bank structure of the laser-cut stent may have exerted an anti-migration effect in situations where the stent was difficult to fully expand due to compression by a growing tumor. However, in a situation where the tumor had shrunk, and the stent was more likely to fully expand, the flared, uncovered end of the braided-type stent may have been more likely to be embedded into the bile duct, making migration less likely.

In fact, some reports have suggested that stent migration may be related to the mechanical properties of metallic stents and the influence of chemotherapy. ${ }^{40,41}$ In recent years, advances in chemotherapy for patients with pancreatic carcinoma have occurred, as shown by reports of prolonged prognosis and improved local control. ${ }^{42,43}$ Therefore, the effect of such intensive chemotherapy on the patency of the stent should be considered in future studies. Stent dysfunction is primarily caused by stent migration in both stent types, and controlling migration is the most important factor associated with prolonged stent patency. The fact that both the inner and outer surfaces of this laser-cuttype CMSs were covered with membranes may have also contributed to the high incidence of migration.

On the other hand, in this study, there was no difference in the OS between the laser-cut-type and braided-type groups, although there was a difference in the TRBO. In both stent groups, there was no significant difference in the OS, probably because of appropriate reinterventions, such as additional placement or replacement of stents even when RBO occurred and continuation of chemotherapy for as long as possible. However, from the perspective of quality of life, the braided-type partially covered SEMSs is superior because it reduces the number of reinterventions. In addition, further development of chemotherapy may prolong the prognosis of patients with pancreatic carcinoma, and stents with longer TRBO enable continued chemotherapy, which may affect the OS in the future.

Our study had certain limitations. First, the study population was relatively small. If the population had been larger, a significant difference in the incidence of stent migration between the two groups would have possibly been observed. However, we used the same stent consecutively for a certain period in the retrospective comparative cohort study. We believe that the statistical reliability should be high in general because most patient background factors were eventually aligned, and no significant differences were observed between the two groups. Second, this study only compared one type of laser-cut CMSs and one type of braided CMSs, which limits the generalizability of the study findings to other types of biliary SEMSs.

Our study is the first to compare laser-cut-type CMSs and braided-type CMSs, both of which have anti-migration systems. In this study, no significant differences in safety and the median OS were observed between the laser-cut CMSs and braided CMSs. However, the TRBO was longer for the braided-type CMSs than for the laser-cut-type CMSs. The braided-type CMSs with an anti-migration system would be preferable for palliation of patients with MDBO due to pancreatic carcinoma. Our results should be corroborated by additional studies. However, stent migration is the major cause of stent dysfunction even in CMSs with anti-migration systems, and controlling migration is of paramount importance for prolonged stent patency.

\section{Conflicts of Interest}

The authors have no potential conflicts of interest.

\section{Funding}

None.

\section{Author Contributions}

Conceptualization: KKi, AM, KS; Data curation: KKi, AM, KS, HK, KM; Formal analysis: KKi, TA, NN, TN, KKa, MF, HT, TO, YF, YS, HY; Methodology: KKi, AM, KS; Writing-original draft: KKi; Writing-review \& editing: all authors. All authors read and approved the final copy of the manuscript.

\section{ORCID}

Koh Kitagawa

Akira Mitoro

Takahiro Ozutsumi https://orcid.org/0000-0001-5794-1512 https://orcid.org/0000-0002-6666-5633 https://orcid.org/0000-0002-6939-0305 


Masanori Furukawa
Yukihisa Fujinaga
Kenichiro Seki
Norihisa Nishimura
Yasuhiko Sawada
Kosuke Kaji
Hideto Kawaratani
Hiroaki Takaya
Kei Moriya
Tadashi Namisaki
Takemi Akahane
Hitoshi Yoshiji

https://orcid.org/0000-0002-9317-9083 https://orcid.org/0000-0003-2402-574X https://orcid.org/0000-0002-5099-2015 https://orcid.org/0000-0002-6295-3283 https://orcid.org/0000-0002-4875-5360 https://orcid.org/0000-0002-1822-6759 https://orcid.org/0000-0002-4361-0592 https://orcid.org/0000-0002-4990-7573 https://orcid.org/0000-0002-2878-8296 https://orcid.org/0000-0002-3158-5318 https://orcid.org/0000-0002-6675-0475 https://orcid.org/0000-0002-5243-8544

\section{REFERENCES}

1. Soehendra N, Reynders-Frederix V. Palliative bile duct drainage: a new endoscopic method of introducing a transpapillary drain. Endoscopy 1980;12:8-11.

2. Huibregtse K, Tytgat GN. Palliative treatment of obstructive jaundice by transpapillary introduction of large bore bile duct endoprosthesis. Gut 1982;23:371-375.

3. Walta DC, Fausel CS, Brant B. Endoscopic biliary stents and obstructive jaundice. Am J Surg 1987;153:444-447.

4. Speer AG, Cotton PB, MacRae KD. Endoscopic management of malignant biliary obstruction: stents of 10 French gauge are preferable to stents of 8 French gauge. Gastrointest Endosc 1988;34:412-417.

5. Huibregtse K, Cheng J, Coene PP, et al. Endoscopic placement of expandable metal stents for biliary strictures: a preliminary report on experience with 33 patients. Endoscopy 1989;21:280-282.

6. Davids PH, Groen AK, Rauws EA, et al. Randomised trial of self-expanding metal stents versus polyethylene stents for distal malignant biliary obstruction. Lancet 1992;340:1488-1492.

7. Knyrim K, Wagner HJ, Pausch J, et al. A prospective, randomized, controlled trial of metal stents for malignant obstruction of the common bile duct. Endoscopy 1993;25:207-212.

8. Lammer J, Hausegger KA, Fluckiger F, et al. Common bile duct obstruction due to malignancy: treatment with plastic versus metal stents. Radiology 1996;201:167-172.

9. Prat F, Chapat O, Ducot B, et al. A randomized trial of endoscopic drainage methods for inoperable malignant strictures of the common bile duct. Gastrointest Endosc 1998;47:1-7.

10. Isayama H, Yasuda I, Ryozawa S, et al. Results of a Japanese multicenter, randomized trial of endoscopic stenting for non-resectable pancreatic head cancer (JM-test): covered wallstent versus doublelayer stent. Dig Endosc 2011;23:310-315.

11. Isayama H, Komatsu Y, Tsujino T, et al. A prospective randomised study of "covered" versus "uncovered" diamond stents for the management of distal malignant biliary obstruction. Gut 2004; 53:729-734.

12. Kitano M, Yamashita Y, Tanaka K, et al. Covered self-expandable metal stents with an anti-migration system improve patency duration without increased complications compared with uncovered stents for distal biliary obstruction caused by pancreatic carcinoma: a randomized multicenter trial. Am J Gastroenterol 2013; 108:1713-1722.

13. Misra SP, Dwivedi M. Reflux of duodenal contents and cholangitis in patients undergoing self-expanding metal stent placement. Gastrointest Endosc 2009;70:317-321.

14. Okamoto T, Fujioka S, Yanagisawa S, et al. Placement of a metallic stent across the main duodenal papilla may predispose to cholangitis. Gastrointest Endosc 2006;63:792-796.

15. Kin T, Ishii K, Okabe Y, et al. Feasibility of biliary stenting to distal malignant biliary obstruction using a novel designed metal stent with duckbill-shaped anti-reflux valve. Dig Endosc 2021;33:648-655.

16. Kogure H, Ryozawa S, Maetani I, et al. A prospective multicenter study of a fully covered metal stent in patients with distal malignant biliary obstruction: WATCH-2 study. Dig Dis Sci 2018;63: $2466-2473$.

17. Park DH, Kim MH, Choi JS, et al. Covered versus uncovered wallstent for malignant extrahepatic biliary obstruction: a cohort comparative analysis. Clin Gastroenterol Hepatol 2006;4:790-796.

18. Isayama $\mathrm{H}$, Mukai $\mathrm{T}$, Itoi $\mathrm{T}$, et al. Comparison of partially covered nitinol stents with partially covered stainless stents as a historical control in a multicenter study of distal malignant biliary obstruction: the WATCH study. Gastrointest Endosc 2012;76:84-92.

19. Kim JY, Ko GB, Lee TH, et al. Partially covered metal stents may not prolong stent patency compared to uncovered stents in unresectable malignant distal biliary obstruction. Gut Liver 2017;11:440-446.

20. Conio M, Mangiavillano B, Caruso A, et al. Covered versus uncovered self-expandable metal stent for palliation of primary malignant extrahepatic biliary strictures: a randomized multicenter study. Gastrointest Endosc 2018;88:283-291.e3.

21. Kullman E, Frozanpor F, Soderlund C, et al. Covered versus uncovered self-expandable nitinol stents in the palliative treatment of malignant distal biliary obstruction: results from a randomized, multicenter study. Gastrointest Endosc 2010;72:915-923.

22. Telford JJ, Carr-Locke DL, Baron TH, et al. A randomized trial comparing uncovered and partially covered self-expandable metal stents in the palliation of distal malignant biliary obstruction. Gastrointest Endosc 2010;72:907-914.

23. Yang MJ, Kim JH, Yoo BM, et al. Partially covered versus uncovered 
self-expandable nitinol stents with anti-migration properties for the palliation of malignant distal biliary obstruction: a randomized controlled trial. Scand J Gastroenterol 2015;50:1490-1499.

24. Ung KA, Stotzer PO, Nilsson A, et al. Covered and uncovered self-expandable metallic Hanarostents are equally efficacious in the drainage of extrahepatic malignant strictures: results of a double-blind randomized study. Scand J Gastroenterol 2013;48:459-465.

25. Costamagna G, Tringali A, Reddy DN, et al. A new partially covered nitinol stent for palliative treatment of malignant bile duct obstruction: a multicenter single-arm prospective study. Endoscopy 2011;43:317-324.

26. Gomez-Oliva C, Guarner-Argente C, Concepcion M, et al. Partially covered self-expanding metal stent for unresectable malignant extrahepatic biliary obstruction: results of a large prospective series. Surg Endosc 2012;26:222-229.

27. Soderlund C, Linder S, Bergenzaun PE, et al. Nitinol versus steel partially covered self-expandable metal stent for malignant distal biliary obstruction: a randomized trial. Endoscopy 2014;46:941-948.

28. Yokota Y, Fukasawa M, Takano S, et al. Partially covered metal stents have longer patency than uncovered and fully covered metal stents in the management of distal malignant biliary obstruction: a retrospective study. BMC Gastroenterol 2017;17:105.

29. Isayama H, Nakai Y, Toyokawa Y, et al. Measurement of radial and axial forces of biliary self-expandable metallic stents. Gastrointest Endosc 2009;70:37-44.

30. Isayama H, Hamada T, Yasuda I, et al. TOKYO criteria 2014 for transpapillary biliary stenting. Dig Endosc 2015;27:259-264.

31. ASGE Standards of Practice Committee, Chandrasekhara V, Khashab MA, et al. Adverse events associated with ERCP. Gastrointest Endosc 2017;85:32-47.

32. Eisenhauer EA, Therasse P, Bogaerts J, et al. New response evaluation criteria in solid tumours: revised RECIST guideline (version 1.1). Eur J Cancer 2009;45:228-247.

33. Kanda Y. Investigation of the freely available easy-to-use software "EZR" for medical statistics. Bone Marrow Transplant 2013;48: 452-458.
34. Yokoe M, Hata J, Takada T, et al. Tokyo guidelines 2018: diagnostic criteria and severity grading of acute cholecystitis (with videos). J Hepatobiliary Pancreat Sci 2018;25:41-54.

35. Isayama H, Kawakubo K, Nakai Y, et al. A novel, fully covered laser-cut nitinol stent with antimigration properties for nonresectable distal malignant biliary obstruction: a multicenter feasibility study. Gut Liver 2013;7:725-730.

36. Saito K, Nakai $\mathrm{Y}$, Isayama $\mathrm{H}$, et al. A prospective multicenter study of partially covered metal stents in patients receiving neoadjuvant chemotherapy for resectable and borderline resectable pancreatic cancer: BTSNAC study. Gut Liver 2021;15:135-141.

37. Marui S, Uza N, Yamazaki H, et al. Utility of laser-cut covered self-expandable metal stents for unresectable malignant distal biliary obstruction: a single-center experience. Endoscopy 2020;52:664-668.

38. Park DH, Lee SS, Lee TH, et al. Anchoring flap versus flared end, fully covered self-expandable metal stents to prevent migration in patients with benign biliary strictures: a multicenter, prospective, comparative pilot study (with videos). Gastrointest Endosc 2011;73: 64-70.

39. Minaga K, Kitano M, Imai H, et al. Evaluation of anti-migration properties of biliary covered self-expandable metal stents. World J Gastroenterol 2016;22:6917-6924.

40. Nakai $Y$, Isayama $H$, Mukai $T$, et al. Impact of anticancer treatment on recurrent obstruction in covered metallic stents for malignant biliary obstruction. J Gastroenterol 2013;48:1293-1299.

41. Nakai $Y$, Isayama H, Kogure H, et al. Risk factors for covered metallic stent migration in patients with distal malignant biliary obstruction due to pancreatic cancer. J Gastroenterol Hepatol 2014;29: $1744-1749$.

42. Conroy T, Desseigne F, Ychou M, et al. FOLFIRINOX versus gemcitabine for metastatic pancreatic cancer. N Engl J Med 2011;364 $1817-1825$.

43. Von Hoff DD, Ervin T, Arena FP, et al. Increased survival in pancreatic cancer with nab-paclitaxel plus gemcitabine. N Engl J Med 2013;369:1691-1703. 